\title{
Morphological Changes in Breast Tissue with Menstrual Cycle
}

Rathi Ramakrishnan, M.D. (Path.), Seema A. Khan, M.D., Sunil Badve, M.D., F.R.C.Path.

Departments of Surgery (RR, SAK) and Pathology (SB), Northwestern University Medical School, Chicago, Illinois and Department of Pathology, Indiana University School of Medicine (SB), Indianapolis, Indiana

Whether the breast tissue undergoes morphologic changes in relation to the menstrual cycle had been a subject of debate. Elegant studies performed in the early 1980s provided conclusive evidence of cyclical changes in the normal breast lobules. These studies were almost entirely based on autopsy material and have not been validated in the clinical setting. In the present study, we examine breast tissues from surgical specimens from 73 premenopausal women and use morphological criteria to characterize the stage of the menstrual cycle. Patients taking oral contraceptives or hormonal therapy were excluded from this study. The following histological parameters were used to assess the menstrual stage: number of cell layers in the acini and presence and degree of vacuolation of the myoepithelial cells, stromal edema, infiltrate, mitosis, and apoptosis. The morphological stage was then correlated with the stage of the cycle, as determined by last menstrual period and the usual menstrual cycle length and in some patients with serum estrogen and progesterone levels. The morphologic stage was concordant with dates in 54 of the 73 patients (74\%, $P=.001)$. In 31 of these patients, serum levels of estradiol and progesterone at the time of surgery were available for correlation. Twenty-five $(80 \%)$ of these were phase concordant by morphology and progesterone levels $(P=.01)$, and $25(80 \%)$, by dates and progesterone levels $(P=.007)$. Women with a high morphologic score were seven times as likely to be in luteal phase as were women with a low score (odds ratio, $7.1 ; 95 \%$ confidence interval). Menstrual phase can be determined by the morphology of the normal lobules present within the surgically excised breast specimens. This will permit

Copyright (C) 2002 by The United States and Canadian Academy of Pathology, Inc.

VOL. 15, NO. 12, P. 1348, 2002 Printed in the U.S.A.

Date of acceptance: September 6, 2002.

Address reprint requests to: Sunil Badve, M.D., F.R.C.Path., Department of Surgical Pathology, University Hospital, Room 3465, 550 North University Boulevard, Indianapolis, IN 46202; e-mail: sbadve@iupui.edu; fax: 317-274-5346.

DOI: 10.1097/01.MP.0000039566.20817.46 retrospective analysis of large archival databases to analyze the effect of timing of surgery in relation to menstrual cycle phase. It will also aid the design of epidemiological studies for breast cancer risk assessment.

KEY WORDS: Breast, Menstrual cycle classification, Menstrual morphology.

Mod Pathol 2002;15(12):1348-1356

The normal breast undergoes changes through the menstrual cycle that affect all aspects of breast morphology, protein expression, and cell kinetics. This physiologic cycling appears to be disturbed in women with breast cancer and may reflect a global dysregulation of response to hormonal influences $(1,2)$. These findings lend greater impetus to studies of normal breast physiology and its possible aberrations in women who are at increased risk for cancer. There is a new urgency to this problem because large, well-defined cohorts of women who have undergone breast biopsy are now available, and the emergence of successful chemopreventive options (3-5) has imparted a practical significance to the task of identifying markers of risk and surrogate markers of chemopreventive success in breast cells. The importance of morphological dating of breast in a clinical setting is 2-fold. One, the knowledge of the histological alterations occurring in the breast with the fluctuating hormonal milieu would enable the identification and enrollment of a cyclematched subset in epidemiological studies relating to breast cancer risk. Two, some but not all studies have suggested an additional prognostic benefit for patients if surgery is performed during the luteal half of the menstrual cycle. This issue can be reexamined by retrospective morphologic dating of the breast tissue relative to the menstrual cycle to exclude discrepancies associated with chronological dating and anovulatory cycles (6-8). There is thus a need for a method for assessing breast samples with respect to the menstrual cycle that (1) can be implemented retrospectively, (2) can capture data re- 
garding the ovulatory state of the cycle, and 3) is reliable and reproducible.

As morphological dating previously has been performed on "normal" autopsy or reduction mammoplasty specimens, parameters for assessing menstrual cycle changes in the breast exist. In this study, we modify these parameters to extend their ability to assess menstrual cycle phase in patients undergoing surgery for benign breast diseases.

\section{MATERIALS AND METHODS}

Women were recruited at the Breast Center of Upstate Medical University (Syracuse, NY) for an ongoing case-control study that aims to correlate expression of hormone receptors with proliferation and apoptosis in normal breast. Patients taking oral contraceptives or hormonal therapy were excluded from this study. The patients included in the current analysis are a subset from this study. Detailed obstetrical and menstrual history, including the date of the last menstrual period and the usual length of the menstrual cycle, were obtained before their breast surgery. The expected date of the onset of the next menstrual period was computed from the average length of the cycle and date of the last menstrual period. The day of cycle at the time of breast surgery was then calculated and adjusted to a 28-day cycle, assuming that the luteal phase duration is more or less constant (14 d), using the following formula:

adjusted day of cycle

$=\frac{14 \times \text { day of the cycle at the time of biopsy }}{\text { length of the follicular phase }}$

For example, in a patient who has an average period length of 32 days, the ovulation is estimated to occur around the 19th day, calculated from the last menstrual period (follicular phase length $=$ $18 \mathrm{~d}$, and luteal phase length $=14 \mathrm{~d}$ ). If the date of biopsy falls on the 16th day in this cycle, her day in the present cycle with respect to a 28-day-cycle would be as follows:

$$
\frac{14 \times 16}{18}=12.44 \text { days }
$$

Inclusion for the purpose of this analysis was restricted to 73 women who did not have breast cancer and had at least two normal terminal duct lobular units present in their biopsy material. In 31 of these patients, serum levels of estradiol and progesterone at the time of surgery were available for correlation. Two pathologists (RR and SB) analyzed hematoxylin and eosin-stained breast sections (1 or
2 per case) from these cases. Thirty cases (exploratory set) were initially analyzed with full knowledge of the menstrual dates in order to develop the morphological criteria. These criteria were then applied to the entire set of 73 cases in blinded review.

The lobules from the breast tissue of the patients from the exploratory set were evaluated for the following histological features: epithelial and myoepithelial distinction; the degree and extent of myoepithelial cell vacuolation, stromal edema, and stromal infiltrate; sharpness of luminal border; and eosinophilic secretions within the lumina. These variables were correlated with menstrual dates to generate the final set of criteria to be applied to the entire set.

Follicular and luteal phase was defined by day of the cycle after standardizing to a 28-day cycle using the last menstrual period and average duration of cycle information (see above). The menstrual cycle was divided into four intervals based on the cyclical fluctuations of the estrogen, progesterone, luteal hormone, and follicular stimulating hormone documented in previous studies (9-11) rather than weekly intervals determined by calendar dates. Early follicular ranged from Day 0 to Day 5 and late follicular, from Day 6 to Day 15. Early luteal phase spanned Day 16 to Day 24, whereas late luteal was between Day 25 to Day 28. The statistical significance was examined using the Pearson's correlation test using the Stata program.

\section{RESULTS}

The age range of the population under study was 18 to 53 years, with a mean of 36.6 years. The commonest of the diagnoses was fibrocystic change followed by fibroadenoma.

\section{Exploratory Set}

Thirty patients with accurate menstrual cycle information in relation to the biopsy date were chosen to establish the morphologic criteria. Histological features of the lobules evaluated were epithelial and myoepithelial layer distinction; proportion of myoepithelial cells exhibiting vacuolation; degree of myoepithelial vacuolation; sharpness of luminal border; and presence of eosinophilic luminal secretions, stromal edema, and stromal infiltrate. The presence of apoptotic bodies usually observed toward the basal portion of the luminal cells and mitotic figures toward the luminal aspect in luminal cells were also noted.

Although no single criterion could distinguish follicular from the luteal phase in all cases, there were significant differences. Distinct epithelialmyoepithelial layers, stromal edema, and infiltrate were predominantly seen in the luteal phase. Mito- 
ses were rarely, if ever, noticed in the follicular phase. Both mitoses and apoptotic figures were frequent in the late luteal phase. However, in a small percentage of cases, apoptotic bodies were also noted in the early part of the cycle. Using these morphological features, it was possible to distinguish the 2 phases in all the cases. The sharpness of luminal borders and luminal secretions were not consistently associated with any particular phase and therefore were not used in the final analysis.

The zones of overlap between late luteal and early follicular and the periovulatory (late follicular and early luteal) groups were more difficult to categorize. The relatively greater delineation between the two layers and degree of myoepithelial vacuolation were helpful in distinguishing early luteal from late follicular phase. Mitotic figures and apoptotic bodies being prominent in the late luteal phase served to demarcate the premenstrual from the early follicular phase. However, in a small percentage of cases, apoptotic bodies was also noted in the early part of the cycle.

Table 1 describes a scoring system that we found useful in assessing stage of menstrual cycle. The individual scores of the variables were added to obtain a final score. The final score ranged between 0 and 19 and correlated with the stage of the cycle (see Table 2). The follicular phase is morphologically characterized by minimal to moderate distinction between epithelial and myoepithelial layers, myoepithelial vacuolation, and stromal changes and tended to get a score between 0 and 9. This phase was further divided into early (Stage 1) and late (Stage 2) by the extent of the two-layer distinction and degree of vacuolation within the acini. The scores in Stage 1 were in the 0-5 range, whereas those for Stage 2 were around 6-9. The luteal half of the menstrual cycle showed prominent epithelialmyoepithelial distinction, myoepithelial vacuolation, stromal edema, and infiltrate, and in the latter stages, the presence of mitoses and apoptotic bodies. The presence of mitotic figures, apoptotic bodies, and stromal infiltrate distinguished early (Stage 3) from late (Stage 4) luteal phase. Thus, the scores in Stage 3 were between 10 and 15, whereas those in Stage 4 were between 16 and 19 .
TABLE 2. Distribution of the Individual Phases by Scores and by Days

\begin{tabular}{lccc}
\hline Menstrual Phase & $\begin{array}{c}\text { Scores by } \\
\text { Morphology }\end{array}$ & $\begin{array}{c}\text { Days } \\
\text { (in Menstrual Cycle) }\end{array}$ & $\begin{array}{c}\text { Phase in } \\
\text { Divisions }\end{array}$ \\
\hline Early follicular & $0-5$ & $0-5$ & 1 \\
Late follicular & $6-9$ & $6-15$ & 2 \\
Early luteal & $10-15$ & $16-24$ & 3 \\
Late luteal & $16-19$ & $25-28$ & 4 \\
\hline
\end{tabular}

\section{Verification Set}

Following the definition obtained from the criteria, the original exploratory set was mixed and blinded, and all the 73 cases were scored using the criteria described above. The morphologic stage was correlated with chronological phase of the cycle and (where available) hormone levels at the time of surgery.

Of the 73 cases analyzed, 39 were morphologically classified as follicular phase (Stages 1 and 2), and 34, as luteal (Stages 3 and 4). Of the 39 patients in the follicular phase, 17 were in Stage 1 and 22 were in Stage 2. Of the 34 cases in the luteal phase, 22 were Stage 3, and 12, Stage 4 . By the adjusted dates (see Materials and Methods for details), 39 patients were in the follicular phase and 34 in the luteal phase. Of these 39 patients, 14 were in the early follicular phase $(0-5 \mathrm{~d})$, and 25 were in the late follicular $(6-15 \mathrm{~d})$. Twenty-five of the 34 luteal phase cases were classified as early luteal by dates (16-24 d), and the remaining 9, as late luteal (24-28 d) (Table 3).

\section{Morphological Changes with Menstrual Cycle}

Distinct alterations in the morphology of the lobule and the intralobular stroma of the breast with some degree of overlap were identified in accordance to the phase of the cycle. Interlobular stroma were relatively unaltered in all these phases. We were able to establish definite criteria to define four different stages of the ovulatory cycle. Summarized here is the correlative hormonal response in each of the stages.

\section{Stage 1 (Menstrual Days 0-5)}

Distinction between the epithelial and the myoepithelial layers was not conspicuous (Fig. 1A). The cells

TABLE 1. Morphological Criteria for the Assessment of Menstrual Phase in the Breast Sections

\begin{tabular}{|c|c|c|c|c|}
\hline Criteria & 0 & 1 & 2 & 3 \\
\hline $\begin{array}{l}\text { Epithelial myoepithelial } \\
\text { distinction }\end{array}$ & 2 distinct layers not seen & $\begin{array}{l}2 \text { layers seen in }<30 \% \text { of the } \\
\text { lobule }\end{array}$ & $\begin{array}{l}2 \text { layers seen in } 31-74 \% \\
\text { of the lobule }\end{array}$ & $\begin{array}{l}2 \text { layers seen in }>75 \% \\
\text { of the lobule }\end{array}$ \\
\hline $\begin{array}{l}\text { Myoepithelial vacuolation } \\
\text { in the acini }\end{array}$ & absent & vacuolation in $<30 \%$ of the cells & $\begin{array}{l}\text { vacuolation in } 31-74 \% \\
\text { of the cells }\end{array}$ & $\begin{array}{l}\text { vacuolation in }>75 \% \text { of } \\
\text { the cells }\end{array}$ \\
\hline $\begin{array}{l}\text { Myoepithelial vacuolation } \\
\text { in the lobule }\end{array}$ & absent & $\begin{array}{l}\text { vacuolation in }<30 \% \text { of the } \\
\text { section }\end{array}$ & $\begin{array}{l}\text { vacuolation in } 31-74 \% \\
\text { of the section }\end{array}$ & $\begin{array}{l}\text { vacuolation in }>75 \% \text { of } \\
\text { the section }\end{array}$ \\
\hline Stromal edema & absent & mild & moderate & marked \\
\hline Stromal infiltrate & absent & mild & moderate & marked \\
\hline Apoptosis & absent & occasional, 1 or 2 & frequent, $>3$ & \\
\hline Mitosis & absent & occasional, 1 or 2 & frequent, $>3$ & \\
\hline
\end{tabular}


TABLE 3. Table Showing the Correlation of Morphology with the Adjusted Dates of the Cycle

\begin{tabular}{cccc}
\hline Phase No. & $\begin{array}{c}\text { Menstrual Phase } \\
\text { by Morphology }\end{array}$ & $\begin{array}{c}\text { Number of Patients } \\
\text { in Phase by } \\
\text { Menstrual Cycle } \\
\text { Day }\end{array}$ & $\begin{array}{c}\text { Number of Patients } \\
\text { in Phase by } \\
\text { Hormones }\end{array}$ \\
\hline 1 & 17 & 14 & 19 \\
2 & 22 & 25 & 12 \\
3 & 23 & 25 & 31 \\
4 & 11 & 9 & 3 \\
Total & 73 & 73 & \\
\hline
\end{tabular}

had round nuclei with minimal and lightly stained cytoplasm. Minimal edema and infiltrate in the intralobular stroma were noted, representing the leftover from the previous cycle. Although rare cells can show vacuolation, it is not a feature (Fig. 1B). Sharp luminal borders with eosinophilic intraluminal secretions were common. Apoptosis and mitosis were by and large absent in this phase. Morphologically, 17 cases were included in this stage, of which 14 matched chronologically to the phase of the cycle.

\section{Stage 2 (Menstrual Days 6-15)}

This phase was characterized by an increase in the distinction between the epithelial and myoepithelial layers of the acini (Fig. 2A). Well-formed, doublelayered acini were appreciated within lobules. Similarly, there was an increasing tendency for the acini to show basal layer vacuolation; however, $<30 \%$ of the lobules showed this feature (Fig. 2B). Stromal edema and infiltrate were absent, and mitoses or apoptotic bodies were not seen. Twenty-two cases fit morphologically in this category, all of which matched with the corresponding day in the cycle.

\section{Stage 3 (Menstrual Days 16-24)}

This phase was characterized by larger lobules with increased numbers of terminal duct units, which were lined by two distinct layers of cells. The basal layer showed marked vacuolations with a centrally or apically placed nucleus with clear and at times vesicular cytoplasm (Fig. 3A-B). The epithelial cells were more oval in nature, with basophilic cytoplasm. The interlobular stroma showed increased edema and a mixed inflammatory infiltrate. Mitotic figures were rare and apoptotic cells infrequent. Twenty-three patients were in this category, all of whom corresponded to the phase by dates.

\section{Stage 4 (Menstrual Days 25-28)}

This phase is morphologically characterized by the presence of extensive vacuolation within lobules. The epithelial cells have prominent nuclei with large nucleoli and a distinctive basophilic hue to the cytoplasm (Fig. 4A). The most characteristic feature is frequent mitotic figures, as well as an increase in apoptosis (Fig. 4B), the latter located toward the base of the lobule. There is extensive stromal edema, with an increase in the inflammatory cell population. Eleven patients were in this stage, and 9 of them matched with the corresponding day in the cycle.

\section{Correlation of Morphology with Chronological Phase of Cycle}

\section{Overall correlation}

Morphologically, 54 of the 73 patients (74\%) were concordant when compared with the adjusted day of the ovulatory cycle $(P=.001)$. Twenty-four of the 34 patients $(70.58 \%)$ were concordant for luteal phase when their morphology was compared with the dates of the cycle $(P=.0001)$. Serum hormone levels were obtained at the time of surgery in 31 of these patients. Of these, 12 patients were in the luteal phase of the cycle (serum progesterone of $\geq 3$ $\mathrm{ng} / \mathrm{L}$ ). Nine of these were in the luteal phase by dates, and 8 were in the luteal phase (Stage 3 or 4 ) by morphology. Women with a high score by morphology were seven times more likely to be in the luteal phase as were women with a low score (odds ratio, $7.1 ; 95 \% \mathrm{CI}$ ).

\section{Correlation of individual parameters}

Of the variables under consideration, the criterion that was most predictive of morphology was the epithelial-myoepithelial distinction $(r=0.78)$, followed by myoepithelial vacuolation $(r=0.7)$ and stromal edema $(r=0.6)$.

\section{DISCUSSION}

Hormonally responsive organs such as the breast and endometrium undergo cyclic changes associated with the menstrual cycle. Although cyclic changes in the endometrium have been well documented, whether similar changes occur in the breast was, until recently, a controversial issue. Although Rosenburg (12) in 1922 correlated the histology of the breast and menstrual cycle and described premenstrual lobulo-alveolar progression and postmenstrual regression of the acini, other investigators (13-15) refuted the possibility of any cycle-related changes in the nongravid breast, calling it essentially a resting gland. These conflicts were partly put to rest by the work of Geschickter (16), who, using autopsy and surgical material, defined two phases of the cycling breast tissue in relation to the menstrual cycle. The first, the phase of "regression" from Day 1 to Day 8, is characterized by atrophy of the epithelium, closing of the alveolar lumina, condensation of the intralobular stroma, and an inflammatory infiltrate. The second 


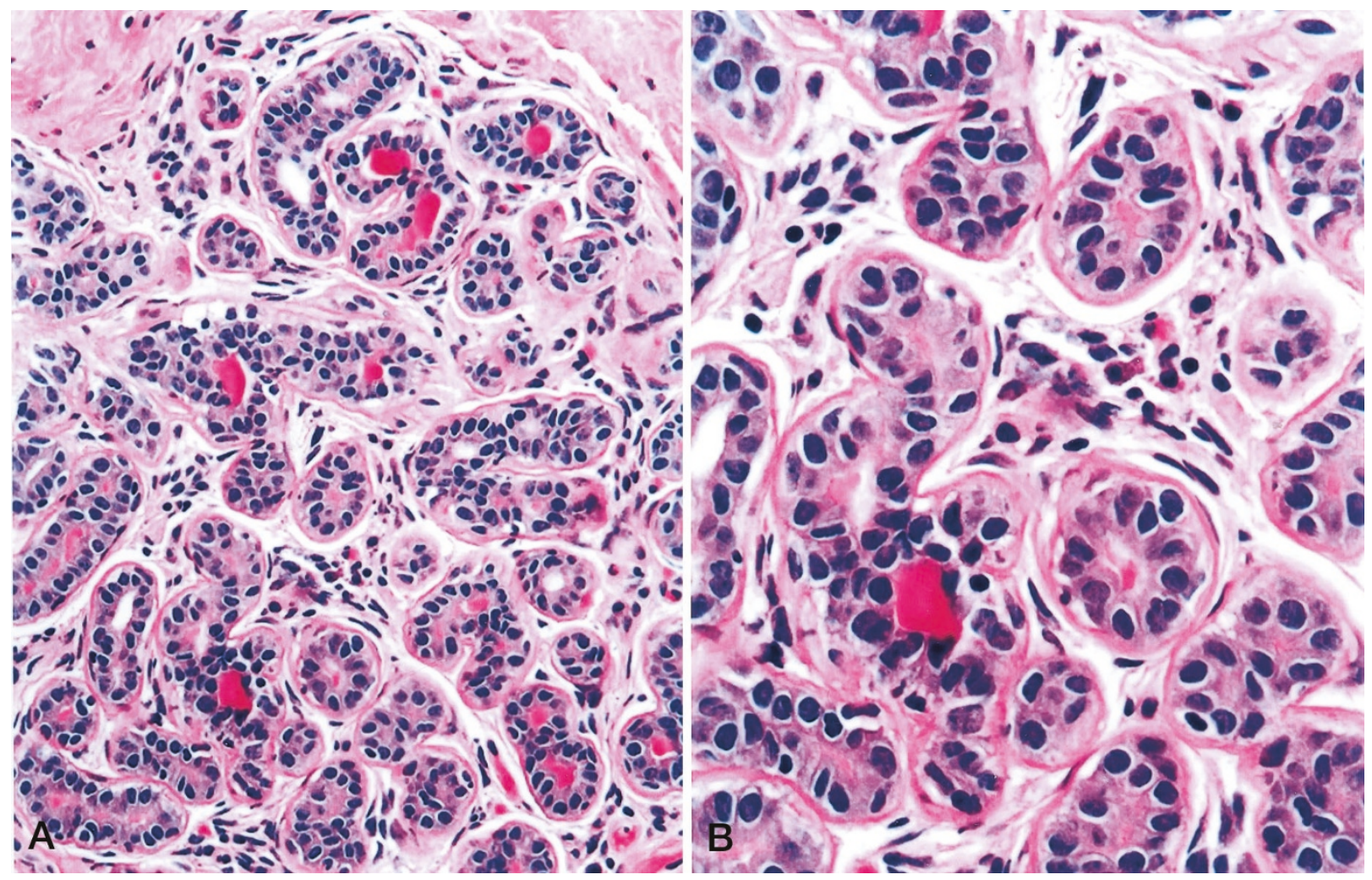

FIGURE 1. Stage 1 of menstrual cycle. A, low magnification of lobule in Stage 1 showing acini clustered with barely perceptible epithelial and myoepithelial layer. Nuclei are round to oval with basophilic cytoplasm and sparse stromal infiltrate, with occasional sprinkling of mast cells seen Eosinophilic secretions are noted within the lumina. H\&E; 100×. B, higher magnification of lobule in Stage 1 showing the uniform lack of vacuolation. H\&E; 400×.

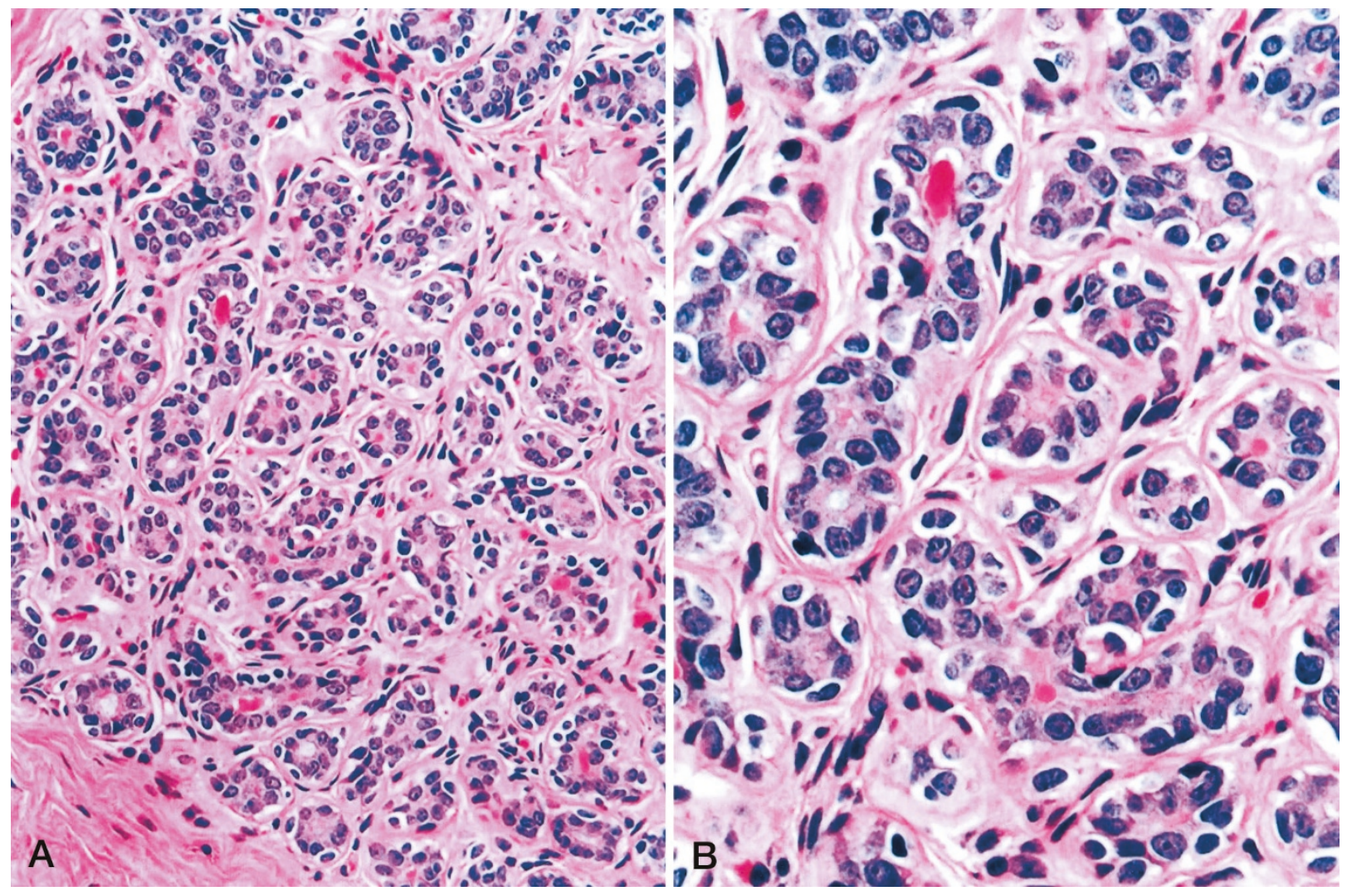

FIGURE 2. Stage 2 of menstrual cycle. A, low magnification of lobule in Stage 2 showing appearance of vacuolation in the myoepithelial layer with prominent distinction between the two layers of the acini. H\&E; 100×. B, high magnification of Stage 2 showing increasing tendency for basal vacuolation. H\&E; 400x. 


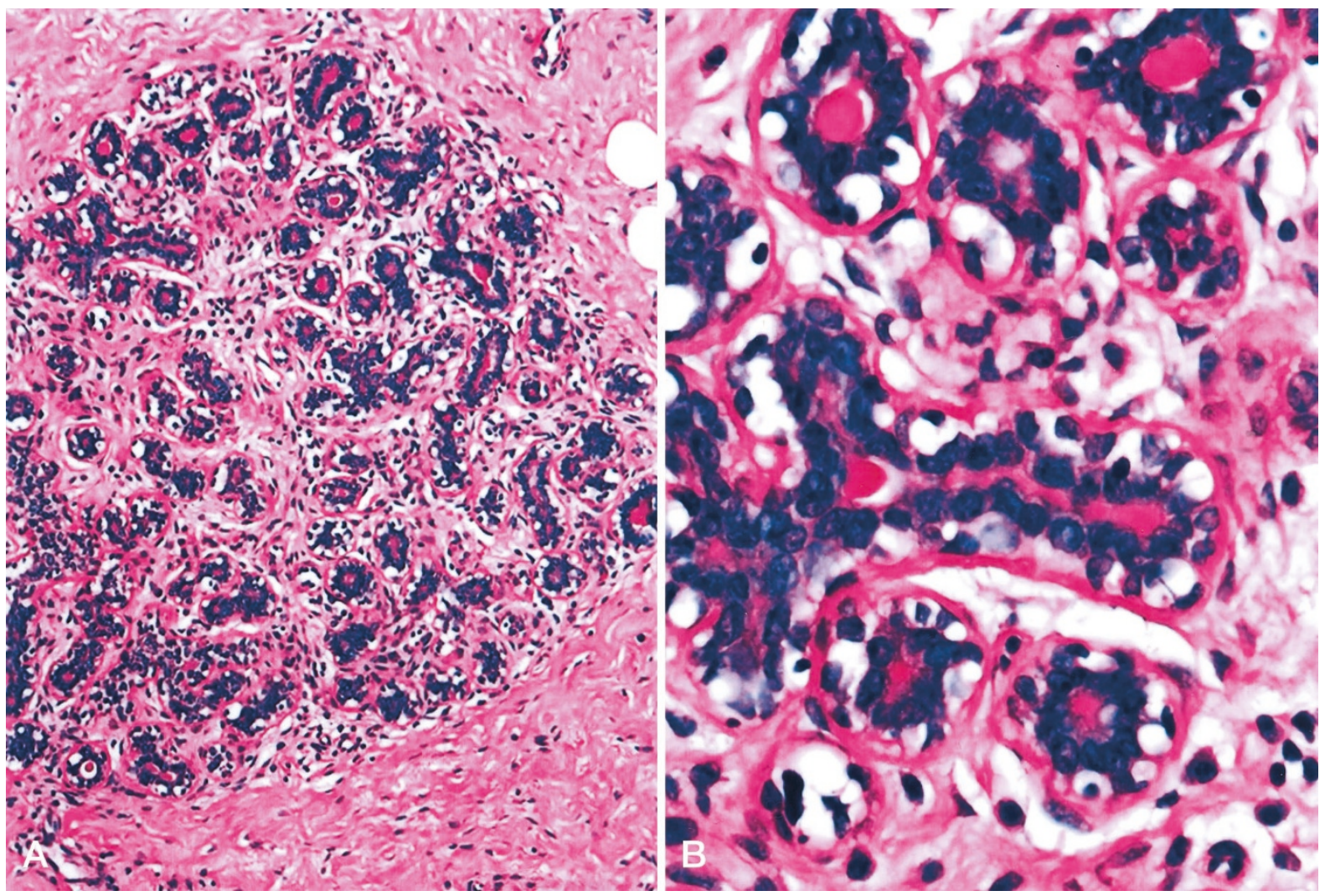

FIGURE 3. Stage 3 of menstrual cycle. A, low magnification of lobule in Stage 3 showing prominent myoepithelial vacuolation with edema of the stroma. The eosinophilic secretions within the lumina are present. H\&E; 100×. B, high magnification of the acini in Stage 3 showing prominent vacuolation. H\&E; 400×.

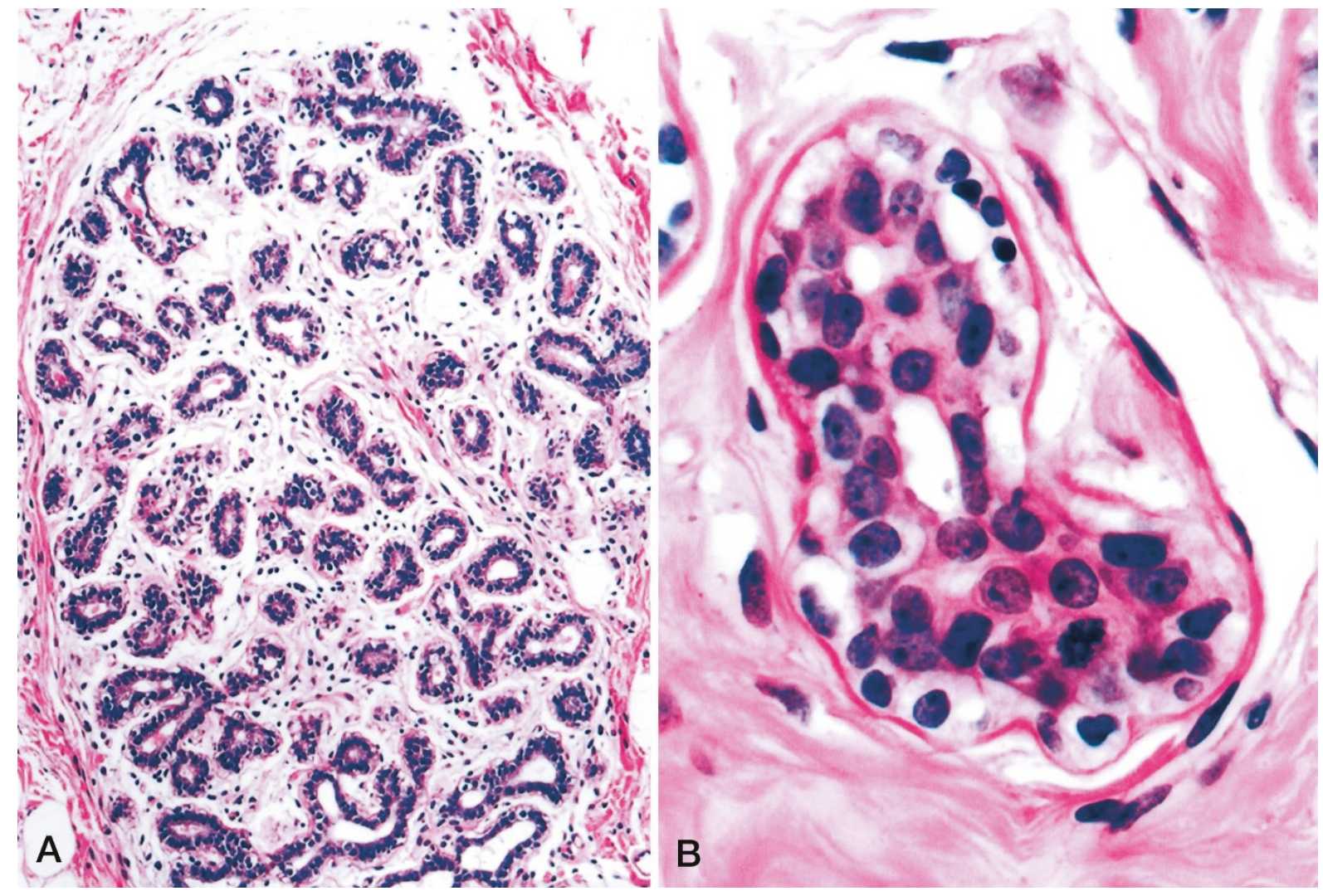

FIGURE 4. Stage 4 of menstrual cycle. A, low magnification of lobule in Stage 4 showing extensive stromal edema and infiltrate with myoepithelial vacuolization. H\&E; 40×. B, high magnification of the acini in Stage 4 showing prominent mitotic figure and apoptotic body in the epithelial layer. H\&E; $400 \times$. 
half was considered proliferative in nature, with alveolar budding, secretions, and stromal edema.

Vogel et al. (11) analyzed nonneoplastic breast tissues from 90 patients undergoing subcutaneous mastectomy and reduction mammoplasty and correlated the histology with menstrual cycle. These patients had no known risk factors for malignancy. Menstrual dates were standardized to a 28-day cycle. The following five phases were identified: proliferative phase, follicular phase of differentiation, luteal phase of differentiation, secretory phase, and menstrual phase. In variance with other studies (17-19), peak mitotic and apoptotic activity in this study were identified in the follicular phase of the cycle. Longacre and Bartow (10) correlated breast and endometrial morphology of tissues obtained at autopsy from 75 premenopausal women who were matched by age and parity status. Size of the lobules, character of the luminal cells, dual lining, myoepithelial vacuolation, intraluminal secretion, stromal edema, mitosis, and apoptosis were used to assess menstrual phase.

Both these studies significantly advanced the knowledge of cyclic changes in menstrual cycle but were never applied in a clinical setup. One of the possible reasons for this not having been attempted to date is illustrated in the work by Foote and Stewart (20) in 1945, wherein they reported a $10 \%$ accuracy in surgically obtained breast tissues. In the same study, a $72 \%$ predictive accuracy for correct identification of the menstrual phase was achieved in autopsy material.

In the current study, we have modified these criteria to correlate cycle status in surgically obtained breast tissue samples. The idea was to devise a reproducible set of criteria that could be applied on breast sections obtained at incisional or core biopsies. This would enable morphological assessment of menstrual status in patients at risk for breast cancer. In these patients, marked alterations and distortion of the lobules is often seen. The lobules with altered morphology, whether due to fibrocystic change or other diseases did not show morphological features that varied with the cycle. All the assessments were, of necessity, made on normal-appearing lobules. As surgical biopsies may have only a small number of "normal" lobular units (or parts thereof) that are available for evaluation, we chose a minimal sample size of two normal lobules to make our modified criteria applicable to most surgical specimens. The limited sample size of the surgical specimens made it impossible to perform the kind of morphometric analysis performed by Longacre and Bartow (10). The current study was performed under these limitations. Morphological criteria were devised using an exploratory set of 30 cases, and their utility was assessed in the entire set of 73 cases.
In the initial exploratory cohort, morphological features such as the distinction between luminal and basal myoepithelial cell layers, degree and proportion of myoepithelial cells vacuolization, character of interlobular stroma, and presence of mitoses and apoptosis were found to be useful in characterizing the phase of menstrual cycle. These parameters were thus used in the latter half of the study. Although no single criterion could distinguish follicular from luteal phase, the appearance of two distinct layers within the acini has proved to be the strongest individual feature distinguishing these two phases of the cycle $(r=0.78)$.

In contrast to the case of the endometrium and in agreement with results of the other morphologic studies (21-25), we found increased mitotic activity in the breast lobules in the luteal phase. Only on rare occasion was a mitotic figure, most likely persistent from the previous cycle, observed in the early follicular phase. This morphologic finding has been confirmed by studies that have compared the tritiated thymidine labeling indices in the follicular and luteal phases (26). The cumulative effect of high levels of estrogen and progesterone that occurs in the late luteal phase $(21,27)$ seems to be necessary to induce proliferation in breast epithelial cells. The proliferative activity appeared confined to the luminal epithelial cells. This finding is supported by experimental data $(9,28)$ that suggest that luminal cells can give rise to both luminal and myoepithelial cells. The breast epithelium, unlike the endometrium, cannot be shed at the end of the cycle; regression at the end of the cycle is by apoptosis. The apoptotic figures are plentiful toward the late luteal phase of the cycle (Stage 4). These findings have been previously described and are supported by quantitative assessments of apoptosis $(21,22)$.

Previous studies that have been based solely on autopsy material have described a good correlation between morphologic phase and chronological phase. However, when applied to surgical material, this correlation was extremely poor (20). In the current study we did not find any morphological parameter in the altered lobules that would allow reliable dating of the breast tissue. However, we found morphologically "normal" lobules within the diseased breast tissue that could be used for dating of the breast tissue. In the current study, we were able to determine phase even when only two lobules were available for assessment. A good correlation between the morphological stage and the chronological phase was obtained when the assessment was restricted to these normal lobules.

The agreement between morphological stage and dates was not complete. The causes of discordance in our study between the two could be due to morphological or chronological factors. Even in the en- 
dometrium, a complete concordance between the two is difficult to obtain, as the menstrual cycle is a continuum of events with degrees of variability between patients as well as between cycles. The chronological dates, although the current gold standard, are a poor standard because they fail to recognize major events such as lack of ovulation, a major reason that endometrial biopsies are performed in patients with infertility. Additionally, as there are wide variations in the cycle length, most studies, including the current one, have to use standardized cycle length. The standardization formulas are based on the assumption that the luteal phase is of fixed duration, although the variability in the duration of the luteal phase has been documented clearly $(7,8)$. These limitations, in our opinion, highlight rather than undermine the importance of morphological evaluation of the phase of menstrual cycle.

The importance of morphological dating of the breast in a clinical setting is 2-fold. A prognostic benefit of timing of surgery during luteal phase of menstrual phase has been seen in some (29-33) but not all studies $(34,35)$. A significant handicap for these studies is the reliability of the menstrual cycle information. Morphological dating of the breast tissue would enable retrospective evaluation of existing large archival databases. Additionally, because the menstrual cycle affects all aspects of breast morphology, protein expression, and cell kinetics, expression of biomarkers must be correlated with the stage of the cycle. Assessment of these markers in large cohorts of patients has been hampered by the lack of accurate menstrual information. The relevance of menstrual cycle in epidemiology and breast cancer risk has not been fully assessed. We hope that dating of breast tissue with regard to menstrual cycle phase will permit (re-)analysis of archival databases and enable us to find answers for these important questions.

\section{REFERENCES}

1. Khan S, Sachdeva A, Naim S, Meguid M, Marx W, Simon H, et al. The normal breast epithelium of women with breast cancer displays an aberrant response to estradiol. Cancer Epidemiol Biomarkers Prev 1999;8:867-72.

2. Khan S, Rogers M, Khurana K, Meguid M, Numann P. Estrogen receptor expression in benign breast epithelium and breast cancer risk. J Natl Cancer Inst 1998;90:37-42.

3. Ingle J. Aromatase inhibition and antiestrogen therapy in early breast cancer treatment and chemoprevention. Oncology (Huntingt) 2001;15:28-34.

4. Shibutani S, Ravindernath A, Terashima I, Suzuki N, Laxmi Y, Kanno Y, et al. Mechanism of lower genotoxicity of toremifene compared with tamoxifen. Cancer Res 2001;61: 3925-31.

5. Fallowfield L, Fleissig A, Edwards R, West A, Powles T, Howell A, et al. Tamoxifen for the prevention of breast cancer: psychosocial impact on women participating in two randomized controlled trials. J Clin Oncol 2001;19:1885-92.
6. Johannisson E, Landgren B, Rohr H, Diczfalusy E. Endometrial morphology and peripheral hormone levels in women with regular menstrual cycles. Fertil Steril 1987;48:401-8.

7. Lenton E, Landgren B, Sexton L. Normal variation in the length of the luteal phase of the menstrual cycle: identification of the short luteal phase. Br J Obstet Gynaecol 1984;91: 685-9.

8. Li T, Rogers A, Lenton E, Dockery P, Cooke I. A comparison between two methods of chronological dating of human endometrial biopsies during the luteal phase, and their correlation with histologic dating. Fertil Steril 1987;48:928-32.

9. Ferguson D. An ultrastructural study of mitosis and cytokinesis in normal "resting" human breast. Cell Tissue Res 1988;252:581-7.

10. Longacre T, Bartow S. A correlative morphologic study of human breast and endometrium in the menstrual cycle. Am J Surg Pathol 1986;10:382-93.

11. Vogel P, Georgiade N, Fetter B, Vogel F, McCarty KJ. The correlation of histologic changes in the human breast with the menstrual cycle. Am J Pathol 1981;104:23-34.

12. Rosenburg A. Ueber Menstruelle durch das corpus luteum bedingte Mammaveranderungen. Frankf Z Pathol Munchen Wiesb 1922;27:466-506.

13. Friedman M, Finkler R, Antopol W. The relation of ovarian hormones to benign breast hyperplasia and neoplasia. Radiology 1939;33:725-36.

14. Haagensen C. Diseases of the breast. 2nd ed. Philadelphia: Saunders; 1971.

15. Taylor H. The relation of chronic mastitis to certain hormones of the ovary and the pituitary and to coincident gynaecological reasons. Surg Gynecol Obstet 1922;62:12948.

16. Geschickter C. Diseases of the breast: diagnosis, pathology, treatment. 2nd ed. Philadelphia: Lippincott; 1945.

17. Ferguson D, Anderson T. Morphological evaluation of cell turnover in relation to the menstrual cycle in the "resting" human breast. Br J Cancer 1981;44:177-81.

18. Going J, Anderson T, Battersby S, MacIntyre C. Proliferative and secretory activity in human breast during natural and artificial menstrual cycles. Am J Pathol 1988;130:193-204.

19. Soderqvist G, Isaksson E, von Schoultz B, Carlstrom K, Tani E, Skoog L. Proliferation of breast epithelial cells in healthy women during the menstrual cycle. Am J Obstet Gynecol 1997;176:123-8.

20. Foote F, Stewart F. Comparative studies of cancerous versus noncancerous breasts. Ann Surg 1945;121:197-222.

21. Anderson T, Ferguson D, Raab G. Cell turnover in the "resting" human breast: influence of parity, contraceptive pill, age and laterality. Br J Cancer 1982;46:376-82.

22. Anderson T, Howell A, King R. Comment on progesterone effects in breast tissue. Breast Cancer Res Treat 1987;10: 65-6.

23. Potten C, Watson R, Williams G, Tickle S, Roberts S, Harris $\mathrm{M}$, et al. The effect of age and menstrual cycle upon proliferative activity of the normal human breast. Br J Cancer 1988;58:163-70.

24. Simpson H, Cornelissen G, Katinas G, Halberg F. Metaanalysis of sequential luteal-cycle-associated changes in human breast tissue. Breast Cancer Res Treat 2000;63:171-3.

25. Speroff L. Role of progesterone in normal breast physiology. J Reprod Med 1999;44:172-9.

26. Meyer J. Cell proliferation in normal human breast ducts, fibroadenomas, and other ductal hyperplasias measured by nuclear labeling with tritiated thymidine. Effects of menstrual phase, age, and oral contraceptive hormones. Hum Pathol 1977;8:67-81.

27. Key T, Pike M. The role of oestrogens and progestagens in the epidemiology and prevention of breast cancer. Eur J Cancer Clin Oncol 1988;24:29-43. 
28. Dulbecco R, Allen W, Bologna M, Bowman M. Marker evolution during the development of the rat mammary gland: stem cells identified by markers and the role of myoepithelial cells. Cancer Res 1986;46:2449-56.

29. Badwe R, Gregory W, Chaudary M, Richards M, Bentley A, Rubens R, et al. Timing of surgery during menstrual cycle and survival of premenopausal women with operable breast cancer. Lancet 1991;337:1261-4.

30. Badwe R, Mittra I, Havaldar R. Timing of surgery during the menstrual cycle and prognosis of breast cancer. J Biosci 2000;25:113-20.

31. Cooper L, Gillett C, Patel N, Bames D, Fentiman I. Survival of premenopausal breast carcinoma patients in relation to menstrual cycle timing of surgery and estrogen receptor/ progesterone receptor status of the primary tumor. Cancer Epidemiol Biomarkers Prev 1999;86:2053-8.
32. Donegan W, Shah D. Prognosis of patients with breast cancer related to the timing of operation. Arch Surg 1993;128: 309-13.

33. Jatoi I. Timing of surgery for primary breast cancer with regard to the menstrual phase and prognosis. Breast Cancer Res Treat 1998;52:217-25.

34. Milella M, Nistico C, Ferraresi V, Vaccaro A, Fabi A, D'Ottavio A, et al. Breast cancer and timing of surgery during menstrual cycle: a 5-year analysis of 248 premenopausal women. Breast Cancer Res Treat 1999;55: 259-66.

35. Nomura Y, Kataoka A, Tsutsui S, Murakami S, Takenaka Y. Lack of correlation between timing of surgery in relation to the menstrual cycle and prognosis of premenopausal patients with early breast cancer. Eur J Cancer 1999;35:132630. 